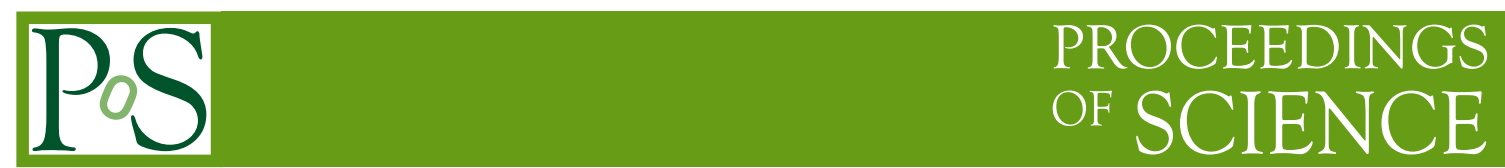

\title{
The equation of state of supernova matter
}

\author{
Abhishek Mukherjee* \\ University of Illinois at Urbana-Champaign \\ E-mail: amukherj@illinois.edu
}

We develop a variational theory of hot dense matter which generalizes the zero temperature variational methods to finite temperatures without any orthogonality corrections. This method can be used to calculate the equation of state for matter in supernovae, protoneutron stars and also heavy-ion collisions using only realistic two nucleon and three nucleon forces as inputs. We present some results obtained with this method for symmetric nuclear matter.

10th Symposium on Nuclei in the Cosmos

July 27 - August 1, 2008

Mackinac Island, Michigan, USA

\footnotetext{
* Speaker.
} 


\section{Introduction}

The equation of state (EOS) of dense matter at finite temperatures is an essential ingredient in the understanding of heavy-ion collision experiments as well as certain astrophysical objects. In the astrophysical context matter inside the iron core of core collapse supernovae reaches supernuclear densities and several tens of $\mathrm{MeV}$ in temperature. The equation of state is thus a vital albeit oftentimes uncertain input into the theoretical modeling of supernovae explosions and the evolution of the protoneutron star subsequently formed.

Although much effort has been devoted to the study of cold (zero temperature) nuclear matter, studies of hot (finite temperature) nuclear matter EOS using realistic potentials have been relatively few. One of the first such calculations was carried out by Friedman and Pandharipande [1] using the variational theory and correlated basis states. Later similar calculations were carried out using a generalization of Brueckner theory to finite temperature via the Bloch-de Dominicis scheme [2]. Recently the method of self consistent Greens functions [3] has also been applied to this problem.

Recently it was shown that although the correlated basis states used in the variational theory are not mutually orthogonal, the free energy when calculated in the variational theory does not have have any orthogonality corrections [4]. It follows immediately that the powerful variational chain summation (VCS) method $[5,6]$ used to derive the Akmal-Pandharipande-Ravenhall zero temperature EOS [7] can be generalized to calculate the EOS of finite temperature nuclear matter without any further approximations. Here we present some results from a much improved variational calculation of the EOS of nuclear matter at finite temperature using this method.

\section{Variational Theory of Hot Dense Matter}

Landau's theory of Fermi liquids assumes that the low lying states of an interacting system, $\Psi_{l}\left[n_{l}(k)\right]$, are in one to one correspondence with those of a noninteracting gas, $\Phi_{l}\left[n_{l}(k)\right]$. In variational theories of quantum fluids one assumes that this mapping is achieved by a symmetrized product of pair correlation operators $\mathscr{F}_{m n}$ [8],

$$
\Psi_{l}\left[n_{l}(k)\right] \propto \mathscr{S}\left(\prod_{m n} \mathscr{F}_{m n}\right) \Phi\left[n_{l}(k)\right]
$$

where $n_{l}(k)$ is the quasiparticle occupation number of the single particle state $k$ and can take the values 0 and 1 for fermionic systems.

By construction, the states $\Psi_{l}$ are not orthonormal to each other.

$$
\left\langle\Psi_{l} \mid \Psi_{l^{\prime}}\right\rangle \neq 0
$$

We need to orthonormalize them before using them in any further calculations

$$
\Psi_{l}\left[n_{l}(k)\right] \stackrel{\text { Orthonormalization }}{\longrightarrow} \Theta_{l}\left[n_{l}(k)\right] .
$$

It is convenient to begin with a Hamiltonian $H_{0}$, defined such that the $\Theta_{l}$ are its eigenstates,

$$
H_{0}=E_{l}^{0} \Theta_{l}
$$


We choose $H_{0}$ such that the eigenvalues $E_{l}^{0}$ can be written as a sum of density $(\rho)$ and temperature (T) dependent quasiparticle energies,

$$
E_{l}^{0}=\sum_{k} \varepsilon(k ; \rho, T) n_{l}(k) .
$$

The quasiparticle entropy is given in terms of the average occupation numbers $\bar{n}(k ; \rho, T)$ by

$$
S_{0}=-k_{B} \sum[\bar{n}(k ; \rho, T) \ln \{\bar{n}(k ; \rho, T)\}+\{1-\bar{n}(k ; \rho, T)\} \ln \{1-\bar{n}(k ; \rho, T)\}] .
$$

Now the thermodynamic variational principle gives an upper bound for the free energy of the system

$$
F \leq\langle H\rangle_{0}-T S_{0}
$$

The right hand side of the inequality is minimized with respect to variations in $\mathscr{F}_{m n}$ and $\varepsilon(k, T, \rho)$ and minimum value is our estimate for the free energy of nuclear matter.

The thermodynamic average of the Hamiltonian is schematically given by

$$
\langle H\rangle_{0} / N=\frac{\hbar^{2}}{2 m} k_{\text {av }}^{2}+\sum \operatorname{diagrams}\left(v, \mathscr{F}, l_{T}\right)+\text { Orthogonality Corrections }
$$

where $N$ is the total number of particles and $k_{\mathrm{av}}^{2}$ is the mean square momentum. The diagrams are many body integrals involving the potential $v$, the correlation function $\mathscr{F}$ and the finite temperature Slater function $l_{T}$. Large classes of diagrams can be summed using the Variational Chain Summation (VCS) method.

The orthogonality corrections on the other hand involve off-diagonal matrix elements in the $\Psi_{l}$ basis, and they cannot be calculated within VCS. In fact there exists no method to calculate the off-diagonal matrix elements and hence the orthogonality corrections accurately. However, it was shown in [4] that there exists an orthonormalization scheme where the orthogonality corrections become vanishingly small in the thermodynamic limit,

$$
\text { Orthogonality Corrections } \longrightarrow 0 \text { when } N \rightarrow \infty .
$$

Thus, the VCS method can be extended to finite temperatures without worrying about orthogonality corrections.

\section{Calculations and Results}

Our correlation operator $\mathscr{F}_{m n}$ reflects the operator structure of the nuclear interactions

$$
\begin{aligned}
\mathscr{F}_{m n} & =\sum_{p=1}^{8} f_{m n}^{p}(r ; \rho, T) O_{m n}^{p} \\
O_{m n}^{p=1-8} & =\left[1, \sigma_{m} \cdot \sigma_{n}, S_{m n}, \mathbf{L}_{m n} \cdot \mathbf{S}_{m n}\right] \times\left[1, \tau_{m} \cdot \tau_{n}\right] .
\end{aligned}
$$

The pair correlation functions $f^{p}(r ; \rho, T)$ and the quasiparticle energies $\varepsilon(k ; \rho, T)$ (assumed to be quadratic in momentum with the bare mass $m$ replaced by an effective mass $m^{\star}$ ) are obtained by minimizing the the free energy at each density and temperature, while simulteneously satisfying the laws of conservation of mass and charge at the level of a few percents. 


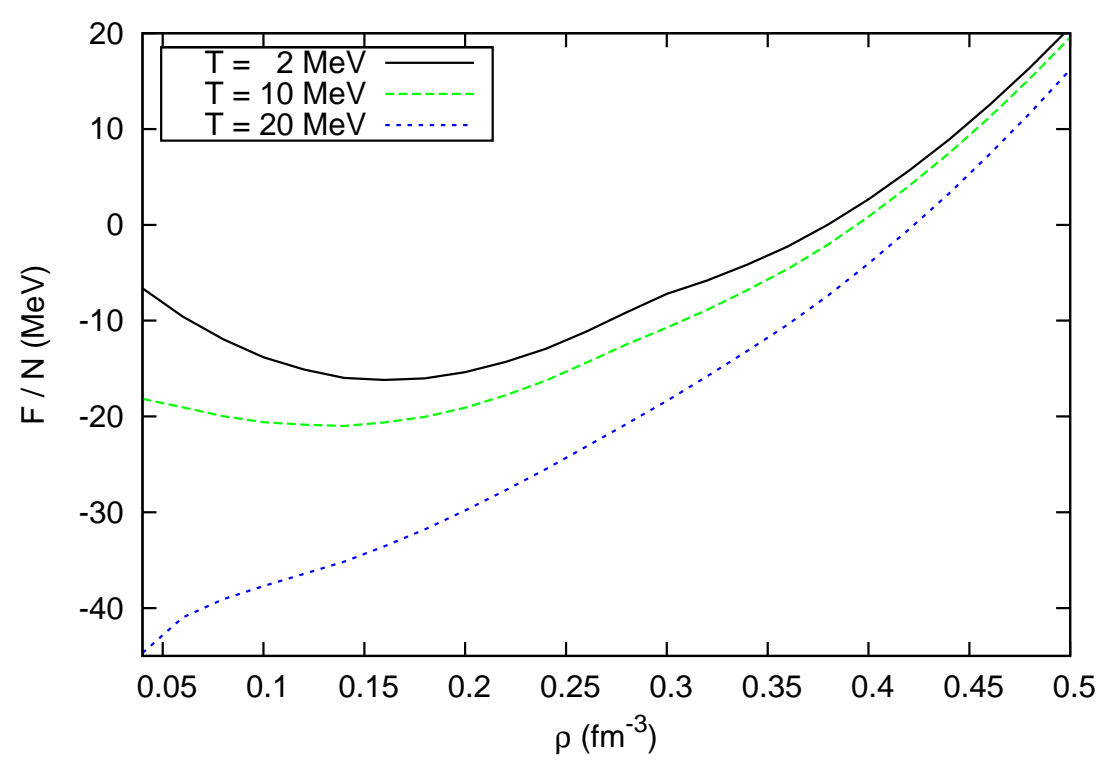

Figure 1: Free energy of symmetric nuclear matter at various temperatures.

We have used the Argonne v18 model for two nucleon interaction [9] and the Urbana IX model [10] of three nucleon interaction. Also the leading order relativistic corrections [11] and the two body perturbative corrections have been added to variational result. In this respect we follow the scheme used by Akmal, Pandharipande and Ravenhall for the zero temperature case [7].

In Fig. (1) the free energy per particle in symmetric nuclear matter for various temperatures is plotted as a function of density. For $T=2 \mathrm{MeV}$, the kink at $\rho \sim 0.30 \mathrm{fm}^{-3}$ is because of the enhanced spin-isospin correlations due to neutral pion condensation [12]. Similar results are also obtained for pure neutron matter.

The pressure of symmetric nuclear matter at $T=10 \mathrm{MeV}$ from the present calculations is plotted in Fig. (2) along with the same from some other commonly used equations of state: FriedmanPandharipande (FP) [1], Lattimer-Swesty (LS) (where $K_{s}$ is the incompressibility) [13] and Shen et al. [14]. It should noted that LS and Shen et al. also consider inhomogeneities for nuclear matter at subsaturation density, while in FP and in the present calculations only a uniform fluid is considered. One can see that there is a noticeable softening of our EOS at $\rho \sim 0.30 \mathrm{fm}^{-3}$ although at higher densities the EOS hardens. Further details can be found in [15].

In conclusion, we have developed a variational theory of hot dense matter which generalizes the variational chain summation method to finite temperatures without any additional corrections. At present we are developing an equation of state which would span wide ranges of temperature, density and asymmetry such that it can be used for modelling of supernova and protoneutron stars.

\section{Acknowledgements}

The many body problem in dense matter was first introduced to the author by the late V. R. Pandharipande. The author is indebted to D. G. Ravenhall and J. Morales for numerous illuminating 


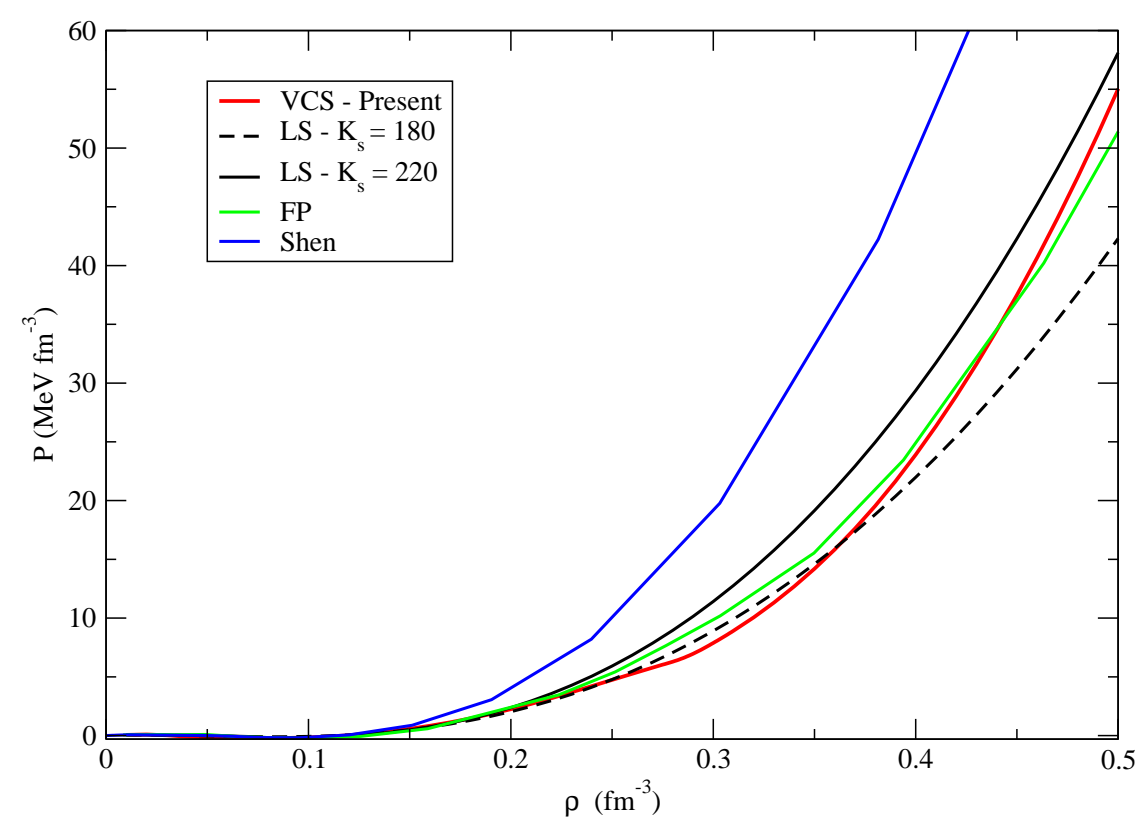

Figure 2: Pressure at $T=10 \mathrm{MeV}$ of symmetric nuclear matter using various methods. See text for details.

discussions. This work was supported in part by US NSF via grant PHY 07-01611.

\section{References}

[1] B. Friedman and V. R. Pandharipande, Nucl. Phys. A 361, 502 (1981).

[2] M. Baldo and L. S. Ferreira, Phys. Rev. C 59, 682 (1999).

[3] A. Rios, A. Polls, A. Ramos and H. Müther, Phys. Rev. C 74, 054317 (2006)

[4] A. Mukherjee and V. R. Pandharipande, Phys. Rev. C 75, 035802 (2007).

[5] V. R. Pandharipande and R. B. Wiringa, Rev. Mod. Phys. 51, 821 (1979).

[6] A. Akmal and V. R. Pandharipande, Phys. Rev. C 56, 2261 (1997).

[7] A. Akmal, V. R. Pandharipande, and D. G. Ravenhall, Phys. Rev. C 58, 1804 (1998).

[8] K. E. Schmidt and V. R. Pandharipande, Phys. Lett. B 87, 11 (1979).

[9] R. B. Wiringa, V. G. J. Stoks, and R. Schiavilla, Phys. Rev. C 51, 38 (1995).

[10] B. S. Pudliner et al., Phys. Rev. C 56, 1720 (1997).

[11] J. L. Forest, V. R. Pandharipande, and J. L. Friar, Phys. Rev. C 52, 568 (1995).

[12] A. B. Migdal, Rev. Mod. Phys. 50, 107 (1978).

[13] J. M. Lattimer and F. D. Swesty, Nucl. Phys. A 535, 331(1992).

[14] H. Shen, H. Toki, K. Oyamatsu, and K. Sumiyoshi, Nucl. Phys. A 637, 435 (1998).

[15] A. Mukherjee, arXiv:0811.3528. 